\title{
Fine-Scale Spatial Response of CdZnTe Radiation Detectors
}

\author{
B.A. Brunett ${ }^{1.3}$, J.M. Van Scyoc ${ }^{1}$, N.R. Hilton ${ }^{1.2}$, J.C. Lund ${ }^{1}$, R.B. James ${ }^{1}$, T.E. Schlesinges
${ }^{3}$ Sandia National Laboratories, Livermore, CA 94550 \\ ${ }^{2}$ Department of Physics, University of Arizona, Tucson, AZ 85724 \\ ${ }^{3}$ ECE Department, Carnegie Mellon University, Pittsburgh, PA 15213
}

\section{Abstract}

Several studies have suggested that the uniformity of Cadmium Zinc Telluride (CZT) detectors play an important role in their performance when operated as gamma-ray spectrometers [1]. However the detailed gamma response of simple planar detectors as a function of position over the device area is largely unknown. To address this issue we have built a system capable of measuring the detector response with a resolution of $\sim 250 \mu \mathrm{m}$. The system consists of a highly collimated $(-200 \mu \mathrm{m})$ photon source $(<150 \mathrm{keV})$ scanned over the detector using a computer controlled, two-axis translation stage. Fifteen samples configured as planar detectors were examined with the new apparatus. The material grade of the detectors examined varied from "counter" to "select discriminator". Two classes of spatial response variation were observed and are presented here. Infrared (IR) transmission images were also acquired for each sample and correlation between features in the pulse height spectrum and crystalline defects were observed.

\section{Introduction}

CZT radiation detector technology has matured rapidly over the last few years. Several new device designs have been demonstrated such as fine pitch imaging arrays [2] and singlecarrier spectrometers [3]. All of these designs require a uniform response over the device area to $\gamma$-irradiation. Therefore it would be useful to be able to measure the spatial variation in the spectral response of commercially available CZT detectors. This system would allow screening of material for some of the novel device designs mentioned above, and for general research into the causes and possible solutions to the uniformity problems. A system has been built to explore these questions in detail. The method we used to analyze the pulse height spectra was very simple. The counts in one or two windows in the pulse height spectrum were evaluated at each position on the sample. More sophisticated analysis could be performed on the pulse height spectrum associated with each spatial point.

\section{Experimental Setup}

\section{A. Equipment}

The mapping system consists of three basic components: a translation mechanism, signal processing and acquisition hardware, and a finely collimated photon source. Pulse height spectroscopy is performed at each spatial point, which gives a local measure of detector performance.
The translation hardware consists of a two-axis stage and motion controller capable of micron resolution. The actuators utilize electrical encoding of the linear output shaft ensuring excellent precision and repeatability.

Pulse height spectroscopy is achieved via a four channel ADC with local histogram memory controlled by a CAMAC dataway. Each channel possesses a dedicated nuclear spectroscopic electronics chain including a shaping amplifier and preamplifier. A pulse height spectrum (PHS) is acquired via the histogram memory for each ADC channel; however, for the results presented in this paper, only a single spectroscopic channel was necessary. A block diagram of the experimental setup is shown in Figure 1.

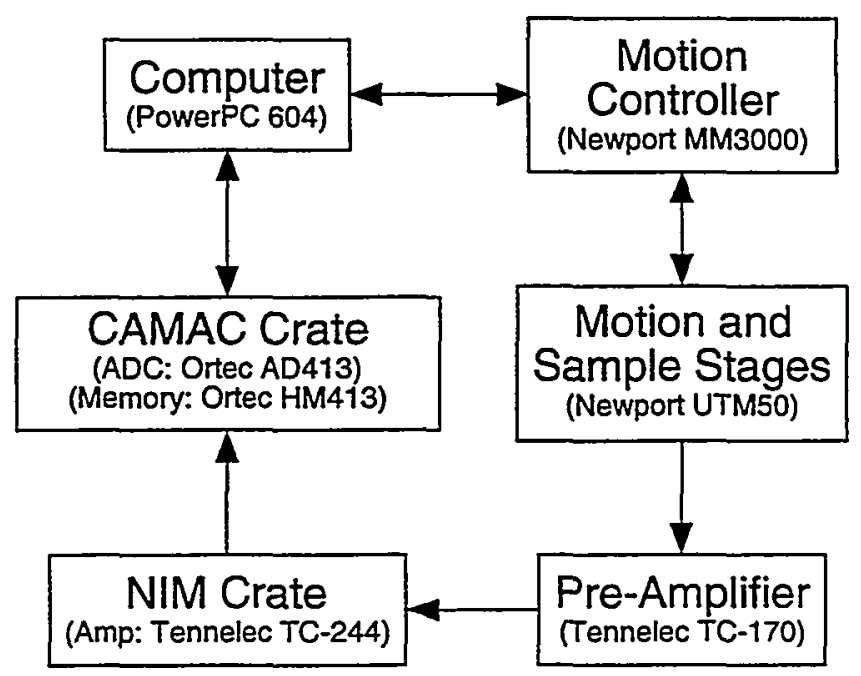

Figure 1: Block diagram showing equipment setup for the mapping system.

The collimator was designed to have a $200 \mu \mathrm{m}$ beam diameter with a working distance of a few $\mathrm{mm}$, and was designed for use with the $122 \mathrm{keV}$ photons from ${ }^{57} \mathrm{Co}$. Unfortunately, ${ }^{57} \mathrm{Co}$ produces a high background due to its $692 \mathrm{keV}$ photon emission. The intensity of this emission line is several orders of magnitude lower than the $122 \mathrm{keV}$ emission but the current configuration of the collimator does not adequately attenuate the high energy photons. This allows the $692 \mathrm{keV}$ photons to flood irradiate the sample while the $122 \mathrm{keV}$ line is limited to a small area.

A second source of significant background illumination was the $\mathrm{Pb}$ fluorescence $(79 \mathrm{keV})$ from the collimator. This photon source was also flood distributed due to the inadequate absorption of the $692 \mathrm{keV}$ photons from ${ }^{57} \mathrm{Co}$. A shield of $\mathrm{Sn} /$ 
$\mathrm{Cu}$ was added to absorb the fluorescence and significantly reduced the $\mathrm{Pb}$ fluorescence peak.

"Mapping is achieved by trans

Mapping is achieved by translating the sample under the $\therefore$ collimated photon source as shown in Figure 2. The photon $\rightarrow$ source remains fixed while the sample, mounting hardware, $\checkmark$ and preamplifier are moved. The sample is configured in a standard planar geometry which requires only two electrical contacts. This configuration eliminates the need for any mechanical access ports allowing a closed sample container which is important for noise considerations. One disadvantage of a planar geometry is that it conceals any information about the spatial distribution of the leakage current or local noise variation.

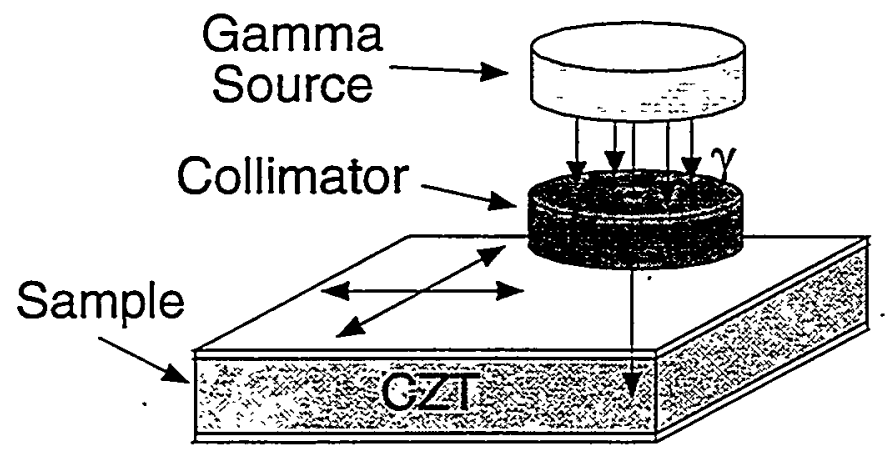

Figure 2: Diagram showing the physical configuration of the sample and collimated ${ }^{57} \mathrm{Co}$ source.

\section{B. Measurements}

Fifteen CZT samples were examined for this study. All samples were tested in a planar configuration and the material grade ranged from "counter" to "select discriminator". Attempts were made to keep the experimental conditions constant for each of the samples examined. The sample bias was chosen to achieve a field of $1000 \mathrm{~V} / \mathrm{cm}$ within the detector. The detector was mounted in a brass sample holder with a Be window and the cathode of the sample was illuminated with the collimated ${ }^{57} \mathrm{Co} \gamma$-source. The spatial response was measured at a resolution of $250 \mu \mathrm{m}$ with an acquisition time of $45 \mathrm{sec}-$ onds per spatial point. The shaping time and gain setting of the shaping amplifier were kept constant for each sample at $1 \mu \mathrm{s}$ and 1000 respectively. The pulse height spectrum acquired for each point was 1024 channels in length and was stored for later analysis.

The data set obtained from the mapping system presented some unique visualization possibilities including both the spatial response and the spectral response. The spatial response is achieved by selecting a channel "window" and then plotting the counts contained within that window for each spatial point in a 2-dimensional map. All of the pulse height spectra from each sample were compressed into 128 channels such that selection of any one of these compressed channels represented a window of 8 channels in the original PHS. The compression was performed to reduce the computational requirements when viewing the data. the count/window representation allows the uniformity of the detector to a particular photon energy range to be examined. The second representation, the spectral response, is useful for determining the spectroscopic capability of a particular region of the detector. This view is achieved by selecting a spatial window and summing the individual PHS obtained at each sampling position into one spectrum representing the region defined by the spatial window. This operation was performed on the original spectra of 1024 channels rather than the compressed spectra to maintain the maximum resolution. The two complimentary representations will be exemplified in the following sections.

Infrared transmission images were also obtained for each sample. After the sample was examined with the mapping system, the contacts were removed and the $\mathbb{R}$ image was taken with a CCD camera. These images were taken to compare the spatial uniformity with any defects observed in the $\mathbb{R}$ images, examples will be shown later in this paper.

\section{Discussion}

\section{A. General}

The spatial uniformity and spectral quality of each sample were examined using a consistent method. A great deal of variation was found both in single samples and between the entire collection of samples. A detailed analysis of one detector will be presented to clarify the analysis methods and the type of results found. A $1 \mathrm{~cm}^{2} \times 2 \mathrm{~mm}$ "counter" was chosen for this example because it showed large variations. The first step in the analysis was to examine the overall $\gamma$-response of the detector. All of the individual PHS were compressed to 128 channels and summed into one spectrum (Figure 3). This spectrum rep-

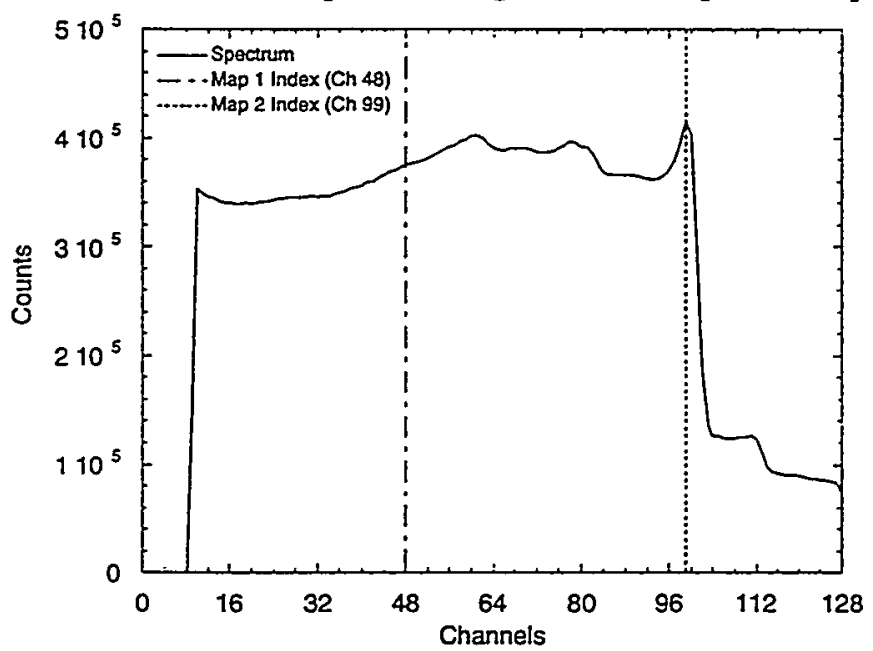

Figure 3: Overall spectral response showing the poor spectroscopic quality of the detector. Also shown are two indexes used to generate spatial response maps.

resents the result that would be obtained if the detector was operated in a standard nuclear spectroscopic system. This detector has very poor spectroscopic performance with a very small photopeak and a very large low energy tail (Figure 3). The next step was to determine if this poor spectroscopic response was due to overall low quality material or if there was a particular region that was degrading the performance. The spatial response was examined to answer this question. Two 


\section{DISCLAIMER}

This report was prepared as an account of work sponsored by an agency of the United States Government. Neither the United States Government nor any agency thereof, nor any of their employees, make any warranty, express or implied, or assumes any legal liability or responsibility for the accuracy, completeness, or usefulness of any information, apparatus, product, or process disclosed, or represents that its use would not infringe privately owned rights. Reference herein to any specific commercial product, process, or service by trade name, trademark, manufacturer, or otherwise does not necessarily constitute or imply its endorsement, recommendation, or favoring by the United States Government or any agency thereof. The views and opinions of authors expressed herein do not necessarily state or reflect those of the United States Government or any agency thereof. 


\section{DISCLAIMER}

Portions of this document may be illegible in electronic image products. Images are produced from the best available original document. 
channels were chosen for analysis and are indicated in Figure 3 as vertical lines. The upper channel was chosen to highlight regions of the crystal that contribute to the photopeak and similarly the lower channel was picked to identify regions giving poor response. From these two channel indices, spatial maps were generated and are given in Figure 4. Two regions

Channel 48
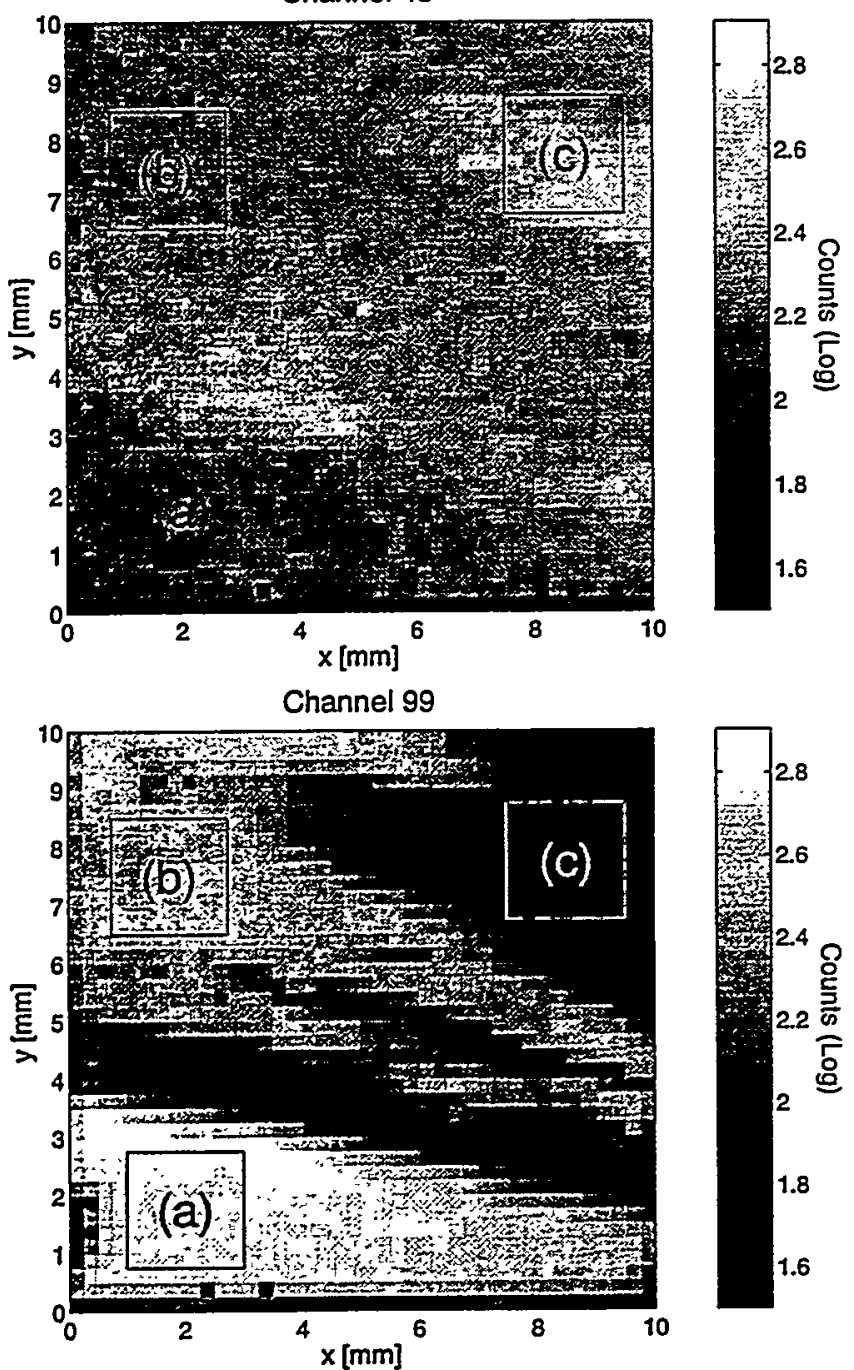

Figure 4: Two-dimensional maps of the spatial response for the two regions indexed in Figure 3. The top map represents the count contribution to the low energy tail and the bottom map shows the counts contributing to the photopeak. The majority of the counts in the photopeak are generated in the lower third of the detector.

can be seen in the top map that contribute a larger number of counts to the lower channels indicating poor response. One spatial region is in the top-right area of the detector and the other runs diagonal near the bottom of the sample. It is likely that these regions give poor spectral response and they will be examined later in this section. The bottom map shows that the majority of the counts in the photopeak were generated in the lower third of the sample suggesting that this region actually has good spectroscopic performance. Three regions were selected to examine the local spectral response indicated as (a), (b), and (c) in Figure 4. Each region was $2 \mathrm{~mm}^{2}$ in area and the individual spectra within the region were summed into one

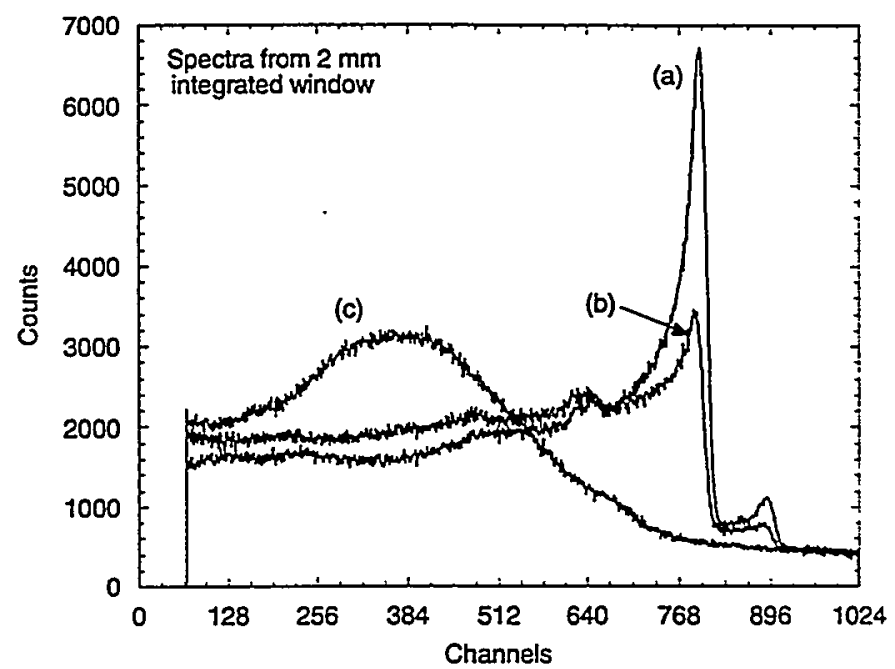

Figure 5: Pulse height spectra resulting from the regions indicated in the spatial response maps of Figure 4 . Notice the complete loss of energy resolution in region (c).

PHS. The spectra resulting from these three areas are shown in Figure 5. Region (c) gives a broad irregular response in which no photopeak is observed. Region (b) exhibits a small photopeak but the energy resolution is poor. And region (a) has respectable spectroscopic response even though the overall quality of the detector was given as "counter". From this analysis it is obvious that the overall poor spectroscopic quality of the entire detector is caused by specific regions within the detector and not by overall poor quality material. It is worth mention that out of all of the samples examined, this "counter" grade detector had one of the best spectral responses when comparing $2 \mathrm{~mm}^{2}$ regions.

\section{B. Defects Diminishing Charge Collection}

The most common feature observed in the samples examined was a region in which the magnitude of the charge collected is reduced from that which is expected. In some instances the pulse height spectrum maintained its shape and was simply shifted to lower channeis (energy). There were also cases in which the shape of the pulse height spectrum was not maintained and the resulting PHS contained a broad irregular peak at low channel numbers. In these cases any energy resolution was completely lost. An example of attenuated charge collection is shown in Figure 6 . The spatial map was generated by selecting a channel index at the shoulder of the PHS attributed to the $122 \mathrm{keV}$ photons. This sample was a $10 \mathrm{~mm}^{2} \times 2 \mathrm{~mm}$ "select counter" grade detector. As in the previous example, this detector also showed a large region that was diminishing the overall energy resolution of the detector. The two regions indicated in Figure 6 give the spectral response plotted in Figure 7.The entire left half of the detector showed reduced charge collection and lower energy resolution than the opposite half of the detector. In addition, the left half of the detector possessed a large density of crystalline defects. These defects can be seen in the IR transmission image of Figure 8. Regions showing reduced charge collection can almost always be linked to the existence of defects within the detector crystal. Previous 
studies suggest that these defects decorate a crystal boundary [4].

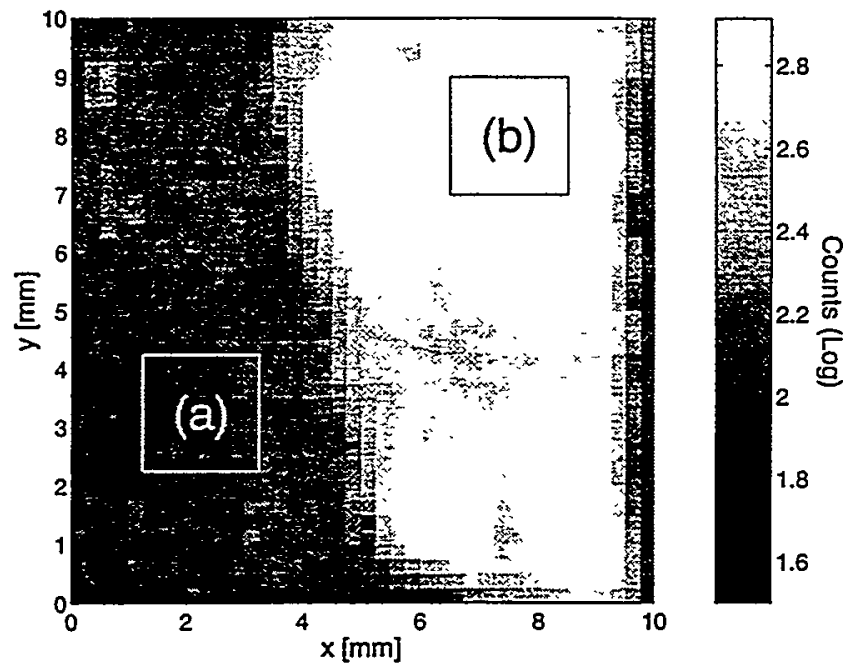

Figure 6: Plot showing the spatial distribution of counts in the photopeak. The $2 \mathrm{~mm}^{2}$ areas indicated were used to examine the spectral response.

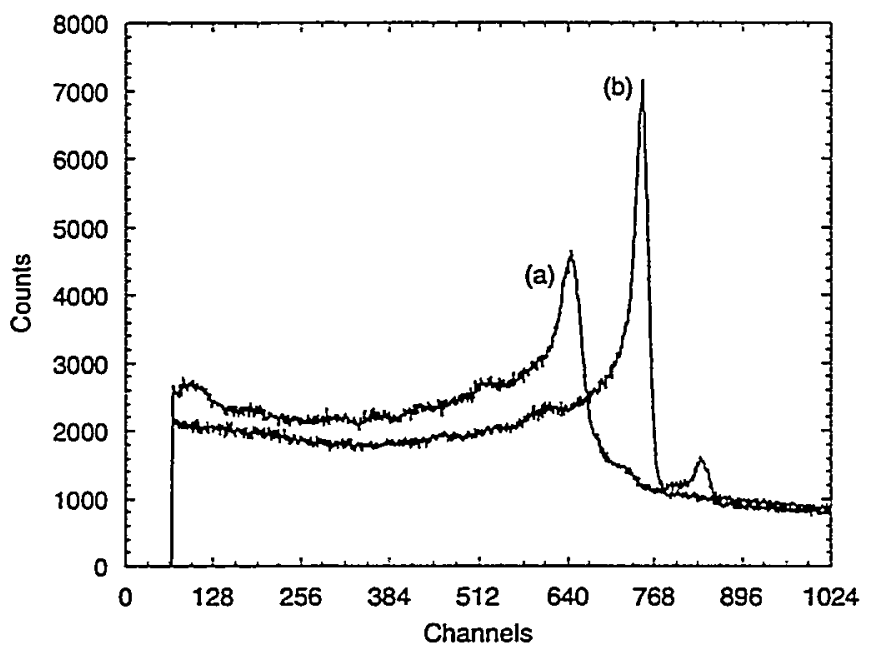

Figure 7: Pulse height spectra resulting from the regions highlighted in Figure 6. Region (a) shows lower charge collection efficiency and energy resolution.

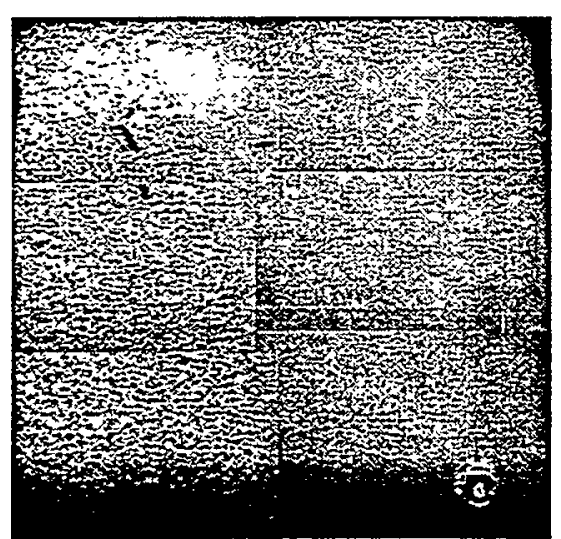

Figure 8: IR transmission image of the detector crystal. A high density of defects can be seen in the left half of the crystal which corresponds to the region with lower charge collection efficiency.

\section{Charge Channeling Defects}

A second type of feature that was observed is the charge channeling feature. The channeling behavior was observed as a region with a deficit of counts relative to other regions of the detector. Unlike the charge reduction features, channeling features did not strongly affect the resulting pulse height spectrum. Additionally, regions of devices that exhibited channeling showed a spatial dependence on the pulse height index used to generate the spatial response map. The channeling feature will shift spatial position as the pulse height index for the spatial map is swept. An example of this type of behavior is shown in Figure 9.
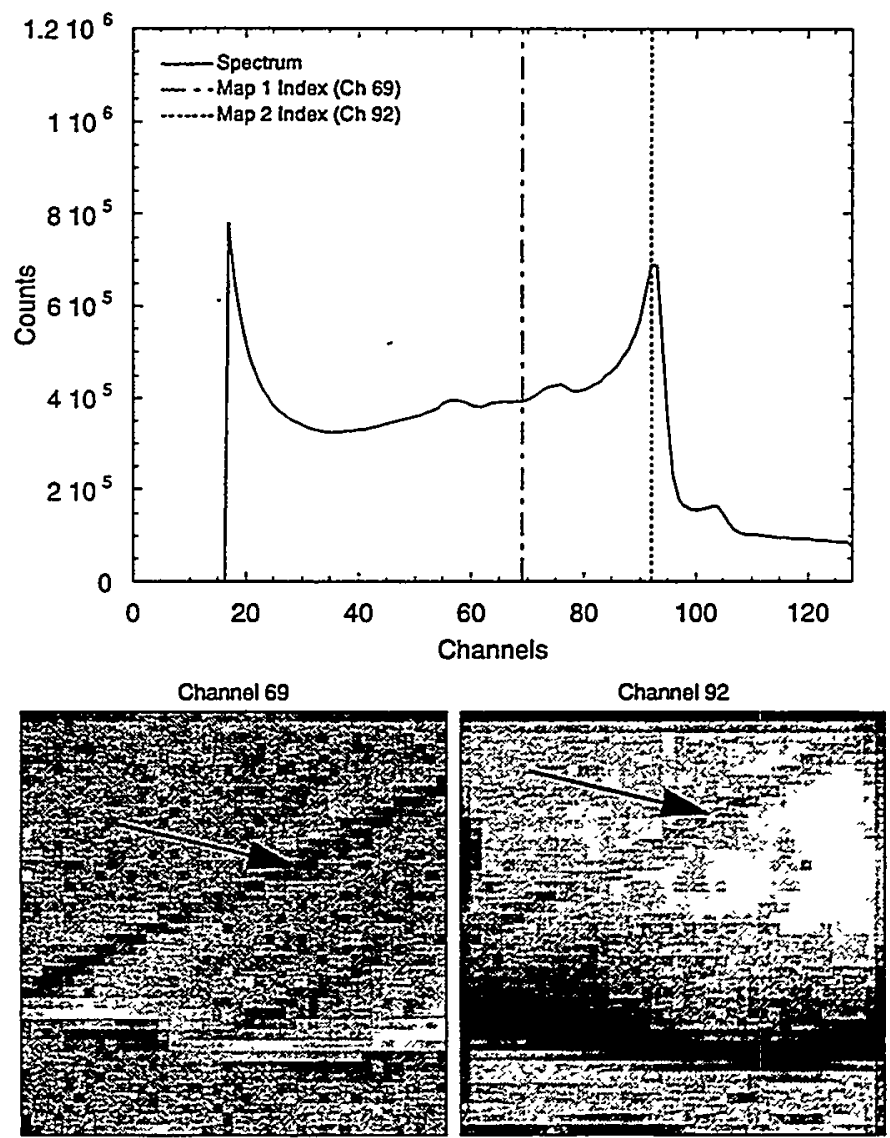

Figure 9: Pulse height spectrum integrated over the detector area (top). Spatial response maps showing the contribution to the photopeak (bottom right) at channel 92, and to $75 \%$ of the photopeak (bottom left) at channel 69. A linear grayscale was applied with black representing the lowest counts. Note the line defect that exhibits a spatial position dependence on the selected channel.

These factors suggest that there is a thin region within the detector that is channeling charge from one position to another. The low energy tail in the CZT pulse height spectrum is due to a interaction position dependence on the collected charge, presumably, by examining successive windows in the pulse height spectrum, we are examining successive slices into the depth of the detector. Therefore, the spatial variation of the channeling feature suggests a planar structure that is inclined to the surface of the detector. Consider the hypothetical example in Figure 10 representing a detector with an electrically active 


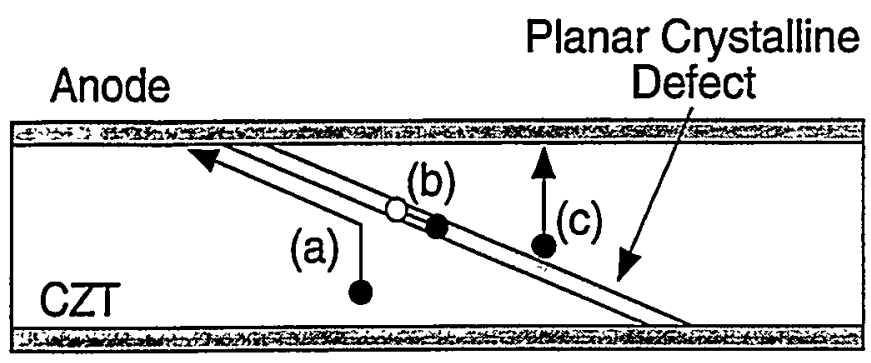

Cathode

Figure 10: Diagram illustrating a hypothetical detector containing a planar crystalline defect. Interactions on the cathode side of the defect (a) will be "channeled" along the defect contributing the expected charge. Interactions on the anode side (c) will be collected normally. Interactions within the defect (b) will become trapped, attenuating the measured charge.

planar defect. Any interactions within the region of the planar defect will be affected, causing a smaller amount of charge to be collected. As we choose different indices in the low energy tail of the pulse height spectrum, we are sampling counts resulting from different interaction depths. Therefore, the spatial position of the observed feature will shift position in the 2dimensional detector response map.

Attempts to find the crystalline defect responsible for this feature were unsuccessful. No crystalline defects could be found in the IR transmission image given in Figure 11. Therefore, it is likely that this type of response is caused by a crystalline defect that is not easily identified by $\mathbb{R}$ transmission analysis. Twinned regions of the crystal are known to be diffcult to detect by IR and might be responsible for the channeling behavior we observed.

A region containing a high density of defects is found where the charge collection is obstructed. This region is indicated by the label (b) in Figure 11 and corresponds to a horizontal band in the lower portion of the spatial response maps.

The dark lines found in the IR image are attributed to defects know as "pipes" which are formed by Ar or Cd vapor bubbles during the growth process. It is believed that they have no effect on detector performance unless they short the detector contacts together [5].

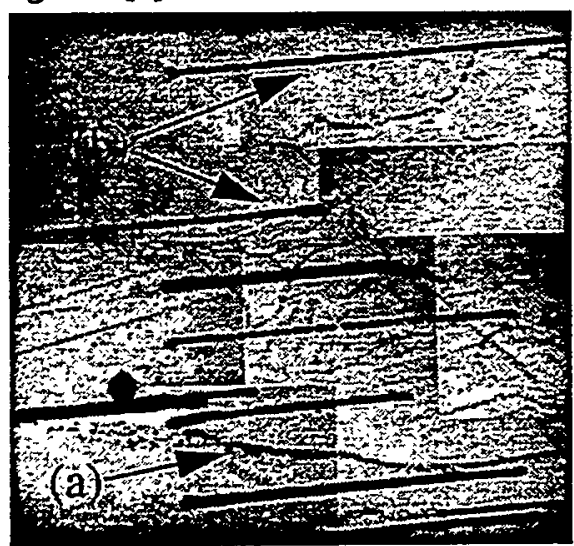

Figure 11: IR transmission image showing an irregular region with a high density of defects (a), several line defects which do not affect the spectral response (b), and the lack of any obvious crystalline defects that may lead to the charge channeling effect.

\section{Conclusion}

The fine-scale spatial response of planar CZT detectors to gamma irradiation has been successfully measured. Large variations in the response have been observed and can be categorized into two classes, charge reduction and charge channeling. Charge reduction is observed as a attenuation in the magnitude of the measured charge from that which is expected. These effects correlate with regions within the detector exhibiting a high density of defects. In some instances this effect is so strong that energy resolution is completely lost. The second variation observed, charge channeling, does not significantly affect the energy resolution for the planar configuration used here. However, a pixellated detector will probably suffer significant performance degradation from a channeling feature. Interactions in one pixel may show up as counts in adjacent pixels [6]. Of the fifteen samples examined three showed a uniform response, three showed the effects of charge channeling, and eleven showed regions of charge reduction. There was no apparent correlation with material grade and spatial uniformity. The strong correlation of crystalline defects with regions of poor charge collection suggest that a simple IR transmission image would be very successful as a screening procedure. Any region containing a large number of defects visible in the image could be avoided when dicing the crystal, allowing a greater yield of higher quality material.

\section{Acknowledgements}

The authors would like to thank Bradford $H$. Parker at NASA Goddard Space Flight Center for obtaining the IR transmission images. We gratefully acknowledge financial support from Sandia's LDRD program (case\#: 3534.020 ). Sandia is a multi-program laboratory operated by Sandia Corporation, a Lockheed Martin Company, for the U.S. Department of Energy under contract number: DE-AC04-94AL85000.

\section{References}

[1] Z. He, "Investigation of Detector Performance of Position Sensitive CdZnTe Detectors," presented at the IEEE Nuclear Science Symposium, Albuquerque, NM, 1997.

[2] K.J. Matherson, et al, "Progress in the Development of Large-Area Modular $64 \times 64 \mathrm{CdZnTe}$ Imaging Arrays for Nuclear Medicine," present at the IEEE Nuclear Science Symposium, Albuquerque, NM, 1997.

[3] P.N. Luke, Single-Polarity Charge Sensing in Ionization Detectors using Coplanar Electrodes," Appl. Phys. Letr., Vol. 65, pp. 2884-2886, 1994.

[4] J.R. Heffelfinger, D.L. Medlin, and R.B. James, "Analysis of Grain Boundaries and Te Precipitates in CdZnTe Grown by High-Pressure Bridgman Method", MRS Symposium Proceedings, Vol. 487, pp. 33-38, 1998.

[5] C. Szeles and E. Eissler, "Current Issues of High-Pressure Bridgman Growth of Semi-Insulating CdZnTe", MRS Symposium Proceedings, Vol. 487, pp. 3-12, 1998.

[6] F.P. Doty, private communication, November 1998. 